\title{
Cumplimiento de la guía clínica de manejo ambulatorio de la neumonía adquirida en la comunidad en personas mayores en centros de atención primaria de salud de Santiago
}

\author{
Hugo Sánchez ${ }^{1}$, Cecilia Albala ${ }^{1}$, Alan D Dangour ${ }^{2}$, \\ Ricardo Uauy 1 . \\ Compliance with guidelines \\ for the management of community \\ acquired pneumonia \\ at primary health care centers
}

Background: Community acquired pneumonia (CAP) has a high mortality rate among older people. To increase its treatment efficiency, the Chilean Ministry of Health elaborated a clinical management guideline for CAP. Aim: To assess the degree of compliance with the clinical guidelines for CAP among professionals working at primary health care centers. Material and methods: A follow up of a cohort of 2,797 subjects aged 67 years or more, incorporated to a clinical study. All cases of bronchopneumonia or pneumonia diagnosed at primary health care centers between September 2005 and June 2008, were recorded. Results: During the follow up period, 192 cases of CAP were diagnosed. A chest X ray was requested in $81 \%$ of cases and a confirmation consultation was done in $58 \%$. Amoxicillin/ Clavulanic acid was the most common antimicrobial prescription in $61 \%$ of cases, followed by Clarithromycin in 17\% and Amoxicillin in 12\%. The antimicrobial used was not registered in $5 \%$ of cases. Conclusions: The clinical guidelines improve the efficiency of CAP treatment and decrease complications. However, these guidelines must be complemented with an adequate training and supervision of health care teams (Rev Méd Chile 2009; 137: 1575-82).

(Key words: Anti-bacterial agents; Pneumonia, bacterial; Standards)

Recibido el 9 de abril, 2009. Aceptado el 18 de noviembre, 2009.

Trabajo Financiado por The Wellcome Trust, Ministerio de Salud Chile.

${ }^{1}$ Unidad de Salud Pública y Nutrición, Instituto de Nutrición y Tecnología en Alimentos, Universidad de Chile. ${ }^{2}$ Nutrition and Public Health Intervention Research Unit, Department of Epidemiology and Population Health, London School of Hygiene \& Tropical Medicine, London, U.K.

Correspondencia a: Dr. Hugo Sánchez R. INTA, Avda. El Líbano 5524, Casilla 138-11, Santiago Chile. Fax: 2214030.

E mail: hsanchez@inta.cl 
$\mathrm{L}$ a neumonía adquirida en la comunidad (NAC) es la principal causa de muerte por infecciones en el mundo ${ }^{1}$. Sus elevadas tasas de incidencia, hospitalización y consumo de servicios de salud, sumado al hecho de constituir una de las patologías que genera más muertes en adultos mayores $^{1,2}$ la transforman en un importante problema de salud pública en este grupo etáreo. Por esta razón, el Ministerio de Salud de Chile incorporó dentro de los objetivos sanitarios para el decenio 2000-2010, la reducción en $20 \%$ de la tasa de mortalidad estandarizada por infecciones respiratorias agudas bajas en los adultos mayores $(\mathrm{AM})^{4}$

La evaluación del progreso en alcanzar estos objetivos a 2004, muestra una reducción de 54\% de la mortalidad por esta causa en adultos de 65 y más años, superando con creces la meta propuesta para el decenio ${ }^{5}$. Elementos claves en el logro de estos buenos resultados, han sido una amplia cobertura de vacunación antiinfluenza y neumocócica, sumada al acceso oportuno a diagnóstico, tratamiento y seguimiento de los $\operatorname{casos}^{6}$.

En 1998, el Ministerio de Salud inicia una serie de innovaciones programáticas a través de la puesta en marcha del Programa Nacional del Adulto Mayor, focalizado en el fomento de un envejecimiento saludable, control y prevención de patologías de gran impacto en este grupo de edad, al cual se suma en el año 2000 al Programa de Enfermedades Respiratorias del Adulto Mayor que incluye como estrategias fundamentales la vacunación anti-influenza, la protocolización del manejo de neumonía y en los últimos tres años la vacunación antineumocócica.

A partir de julio de 2005 el Estado de Chile incorporó al Régimen de Garantías Explícitas en Salud (AUGE) el manejo ambulatorio estandarizado de la NAC de los adultos de 65 y más años ${ }^{7,8}$. Como parte de esto, el Ministerio de Salud elaboró la guía clínica para el manejo de las neumonías en todos los centros de atención primaria (CAP) del país. La guía clínica incluye, además del tratamiento médico, apoyo de kinesiólogo y enfermera dependiendo de la gravedad del cuadro. Además estableció garantías de acceso: a un diagnóstico oportuno, confirmación diagnóstica a través de radiografía de tórax dentro de las primeras $48 \mathrm{~h}$ y tratamiento farmacológico por 10 días.
El desarrollo y cumplimiento de protocolos para el manejo estandarizado de la NAC ha probado mejorar el cuidado y pronóstico de la enfermedad de una manera eficiente ${ }^{1,9,10}$. Sin embargo, la información existente muestra que la adherencia de los médicos a estos protocolos es a menudo subóptima ${ }^{11}$.

El objetivo del presente trabajo es evaluar el nivel de cumplimiento de la Guía Clínica AUGE para el manejo de la NAC de los adultos de 65 y más años, por parte de los equipos de salud de Atención Primaria.

\section{SujETOS Y MÉTODO}

El presente estudio se efectuó en el marco del Proyecto CENEX, uno de cuyos objetivos principales fue estudiar la costo-efectividad del Programa de Alimentación Complementaria para el AM (PACAM) en la incidencia de neumonía. Dicho estudio, cuyo protocolo detallado ya ha sido publicado $^{12}$, se realizó entre los años 2005 y 2008 en 2.797 personas de 65 a 67 años, autovalentes, usuarios del sector público de salud, distribuidos en 28 Centros de Atención Primaria ubicados en el área urbana del Gran Santiago.

El proyecto CENEX buscó determinar si esta intervención nutricional mejora la inmunidad de los AM, lo que se evaluó a través de la incidencia de neumonía en los sujetos de la cohorte. Entre septiembre de 2005 y junio de 2008 se registraron todos los cuadros diagnosticados como bronconeumonía o neumonía y tratados en forma ambulatoria en los CAP de acuerdo a la guía clínica $\mathrm{GES}^{13}$.

Recolección de datos. La recolección de información estuvo a cargo de profesionales contratados por el estudio, previamente capacitados y estandarizados, los que realizaron revisiones periódicas de las fichas clínicas, recetas médicas despachadas en la farmacia del CAP, hojas de registro de atención diaria y registros clínicos de las Salas de Enfermedades Respiratorias del Adulto (Sala ERA). En los casos de neumonía correspondientes a sujetos del estudio CENEX, se registró el diagnóstico en un formulario elaborado para estos fines, consignando las fechas y el cumplimiento de cada una de las prestaciones incluidas en la guía 
clínica: consultas médicas de sospecha y confirmación diagnóstica, solicitud y realización de radiografía de tórax y la descripción del informe radiológico, número de consultas realizadas por enfermera y kinesiólogo, entrega de termómetro, solicitud y realización de hemograma y uremia y el nombre, dosis y duración de los medicamentos utilizados en el tratamiento.

Análisis de datos. Se comparó el grado de cumplimiento de la guía clínica a partir de los datos obtenidos del seguimiento, separando el análisis en: Confirmación diagnóstica, que incluye la indicación de radiografía de tórax y control dentro de las $48 \mathrm{~h}$ y del Tratamiento, el cual incluye tipo y duración del tratamiento indicado y derivación a enfermera o kinesiólogo de los casos. Se consideró como puntos críticos de cumplimiento de la guía clínica, el control a las $48 \mathrm{~h}$, la realización de radiografía de tórax, y el tratamiento antibiótico por diez días, según lo señalado en la guía clínica.

Estadística. Los resultados son expresados como valores promedio y desviación estándar en el caso de las variables continuas con distribución normal y como mediana y rango intercuartílico, en el caso de no presentar normalidad en la distribución. Las variables categóricas se expresan en porcentajes. Se analiza el cumplimiento de las guías según sexo y comuna. Para su análisis estadístico se utilizó el test $\chi^{2}$ estableciéndose un nivel de confianza de 95\%. El análisis de los datos se realizó con el programa STATA 10.1.

\section{Resultados}

Durante el período de seguimiento de los sujetos incluidos en el estudio CENEX, se diagnosticaron 192 casos de neumonía, en 153 adultos mayores (127 personas con 1 caso de neumonía, 19 con 2 casos y 7 con 3 o más casos de neumonía).

El número de casos presentó una alta variabilidad intercentros, con tasas de incidencia entre 5,0 y 65,8 casos por cada 1.000 personas año de seguimiento. En la Tabla 1 se detalla la información epidemiológica básica desagregada por comuna y CAP. De los 192 casos notificados, 10 casos fueron derivados a centros hospitalarios para su tratamiento, los cuales fueron excluidos con el fin de restringir el análisis, al manejo ambulatorio de los casos.

El cumplimiento de la confirmación diagnóstica de acuerdo con la guía AUGE se presenta en la Tabla 2. Se observa que $60,4 \%$ de los casos diagnosticados como neumonía en la primera consulta se les realizó una segunda consulta, de las cuales 51,8\% se efectuó antes de las $48 \mathrm{~h}$. La mediana de tiempo entre la primera consulta y segunda consulta fue de 2 días con un IQR de 3, no observándose diferencias por sexo, ni comuna ( $p=0,52 / 0,09)$. A 82,4\% de los sujetos se les indicó radiografía de tórax, realizándose el examen en $92,0 \%$, con una mediana de 1 día, con un IQR de 2 entre la indicación de radiografía y su realización. No se presentaron diferencias por sexo, ni comuna ( $p=0,92 / 0,30)$

Los resultados pertinentes al tratamiento se presentan en la Tabla 3, observándose que el esquema de tratamiento más frecuente fue la combinación de amoxicilina/ácido clavulánico en $61,2 \%$ de los casos tratados, la segunda opción fue claritromicina con $17,4 \%$ y en tercer orden de uso fue amoxicilina con $14,0 \%$. No se registró el antibiótico indicado en $5,2 \%$ de los casos. La mediana de tratamiento fue de 10 días para los antibióticos descritos.

La utilización de inhalador, que de acuerdo a la guía se indica según criterio médico clínico, se observó en $64,3 \%$ de los casos, con una mediana de tratamiento de 7,3 días y 2,0 inhalaciones diarias cada $4 \mathrm{~h}$. El inhalador recetado más frecuente fue el salbutamol (92,3\%). El uso de otros medicamentos se observó en $63,2 \%$ de los casos, siendo los más frecuentes el acetaminofeno $(60,9 \%)$ y prednisona $(12,2 \%)$.

El uso de recursos profesionales de salud por episodio de neumonía se detalla en la Tabla 4, observándose que sólo $42,8 \%$ tuvo 3 consultas médicas, número indicado como mínimo en la guía clínica AUGE, no existiendo diferencias por sexos $(p=0,06)$ y sí entre las comunas $(p=0,03)$. El 40,1\% de los pacientes tuvo tratamiento kinésico, no existiendo diferencias entre comunas ni sexo. La mediana de atenciones kinésicas fue 2 con un rango de 1 a 7 , sin diferencias entre hombres y mujeres $(p=0,49)$ y con diferencias entre las comunas $(p<0,001)$. Cabe señalar que $100 \%$ de los CAP cuenta con horas profesionales 
Tabla 1. N úmero de casos de neumonía y tiempo de seguimiento por Centros de Atención Primaria, incluidos en el estudio

\begin{tabular}{|c|c|c|c|c|c|}
\hline Comuna & $\begin{array}{l}\text { Centros de } \\
\text { Atención Primaria }\end{array}$ & $\begin{array}{c}\text { Población } \\
65 \text { a } 69 \text { años* }\end{array}$ & $\begin{array}{l}\text { n sujetos } \\
\text { enrolados }\end{array}$ & $\begin{array}{l}\text { Tiempo } \\
\text { de observación } \\
\text { en meses }\end{array}$ & № casos \\
\hline Conchalí & José Symon Ojeda & 1.416 & 115 & 31 & 17 \\
\hline Conchalí & Juanita Aguirre & 922 & 114 & 32 & 3 \\
\hline Conchalí & Eneas Gonel & 945 & 100 & 24 & 8 \\
\hline Estación Central & Cinco & 1.596 & 69 & 34 & 3 \\
\hline La Cisterna & Santa Anselma & 1.219 & 107 & 32 & 5 \\
\hline La Cisterna & Eduardo Frei Montalva & 1.445 & 100 & 24 & 7 \\
\hline La Florida & Bellavista & 2.250 & 115 & 34 & 4 \\
\hline La Florida & Los Castaños & 1.251 & 100 & 31 & 17 \\
\hline La Florida & Los Quillayes & 1.126 & 103 & 31 & 5 \\
\hline La Florida & Maffioletti & 1.763 & 100 & 33 & 6 \\
\hline La Florida & Villa O'Higgins & 1.581 & 106 & 33 & 7 \\
\hline La Granja & La Granja & 1.331 & 100 & 33 & 3 \\
\hline Macul & Félix de Amesti & 2.093 & 101 & 32 & 10 \\
\hline Macul & Santa Julia & 1.794 & 108 & 33 & 3 \\
\hline Peñalolén & San Luis & 1.253 & 101 & 24 & 2 \\
\hline Peñalolén & Lo Hermida & 1.245 & 100 & 24 & 6 \\
\hline Peñalolén & Carol Urzúa & 1.431 & 100 & 24 & 1 \\
\hline Peñalolén & La Faena & 1.563 & 100 & 24 & 5 \\
\hline Quinta Normal & Lo Franco & 805 & 96 & 24 & 8 \\
\hline Quinta Normal & Andes & 610 & 94 & 31 & 12 \\
\hline Quinta Normal & Garín & 2.091 & 114 & 31 & 15 \\
\hline San Joaquín & Arturo Baeza Goñi & 589 & 66 & 34 & 9 \\
\hline San Joaquín & San Joaquín & 914 & 100 & 31 & 13 \\
\hline San Joaquín & Sor Teresa de los Andes & 1.509 & 101 & 32 & 5 \\
\hline San Miguel & Recreo & 957 & 80 & 34 & 3 \\
\hline San Miguel & Barros Luco & 1.202 & 100 & 24 & 1 \\
\hline San Ramón & La Bandera & 1.330 & 99 & 31 & 8 \\
\hline San Ramón & San Ramón & 1.113 & 108 & 31 & 6 \\
\hline Total & & & 2.797 & & 192 \\
\hline
\end{tabular}

*Población inscrita validada a 30 de septiembre 2006. Fuente: FONASA.

de kinesiólogo. La atención por enfermera sólo se observó en 5 casos de sexo femenino.

La solicitud de exámenes de laboratorio se efectuó en 8 casos para hemograma y 3 casos para nitrógeno ureico. En relación a la entrega de termómetro para el control de la temperatura en domicilio, no se indicó en ninguno de los casos estudiados.

\section{DisCUSIÓN}

El proceso de reforma sanitaria iniciado por el Estado de Chile en el año 2002, ha establecido, para un conjunto de patologías, garantías de acceso, de oportunidad, de protección financiera y de calidad, las cuales están incluidas dentro del $\mathrm{AUGE}^{7}$. Los grandes esfuerzos realizados a la 
Tabla 2. Cumplimiento de G uía Clínica AU G E para la confirmación de “N eumonía Adquirida en la Comunidad en manejo ambulatorio en personas de 65 años y más"

\begin{tabular}{|c|c|c|c|}
\hline & $\begin{array}{c}\text { H ombres } \\
n=35\end{array}$ & $\begin{array}{l}\text { M ujeres } \\
n=147\end{array}$ & $\begin{array}{c}\text { Total } \\
n=182\end{array}$ \\
\hline $\begin{array}{l}\text { Consulta de confirmación } \\
p=0,52\end{array}$ & $20(57,1)$ & $90(61,2)$ & $110(60,4)$ \\
\hline $\begin{array}{l}2^{\text {a } \text { Consulta }} \\
<48 \mathrm{~h}\end{array}$ & $11(55,0)$ & $46(51,1)$ & $57(51,8)$ \\
\hline $2^{\text {a }}$ Consulta después de $48 \mathrm{~h}$ & $9(45,0)$ & $44(48,9)$ & $53(48,2)$ \\
\hline Mediana & 2 & 2 & 2 \\
\hline IQR & 2,5 & 3 & 3 \\
\hline Rango & $0-9$ & $0-19$ & $0-19$ \\
\hline $\begin{array}{c}\text { Indicación Rx } \\
p=0,54\end{array}$ & $27(77,1)$ & $123(83,7)$ & $150(82,4)$ \\
\hline Rx realizada & $26(96,37)$ & $112(91,0)$ & $138(92,0)$ \\
\hline Mediana & 0 & 1 & 1 \\
\hline IQR & 2 & 2,5 & 2 \\
\hline Rango & $0-6$ & $0-19$ & $0-19$ \\
\hline
\end{tabular}

fecha han mejorado el acceso a servicios de salud, la oportunidad de la atención y la protección financiera.

La garantía de calidad está hoy en día en pleno proceso de implementación con el inicio de los procesos de acreditación de prestadores de salud. En este sentido, la elaboración y difusión de guías clínicas para el manejo de las patologías incluidas en este régimen, es un gran avance que ha realizado el Ministerio de Salud de Chile.

La optimización del proceso de atención de salud de los casos de NAC a través de guías clínicas actualizadas, mejora su evolución y disminuye las complicaciones asociadas 9,10 . Por ello constituye una importante herramienta para los gestores locales de salud, tanto a nivel hospitalario como de los municipios que administran la atención primaria de salud.

En la actualidad, 320 comunas a nivel nacional administran la salud primaria, gestionando todos ellos uno o más establecimientos de salud, que van desde postas de salud rural a centros de salud familiar. La posibilidad de contar con herramientas de apoyo a la gestión clínica en los municipios es de gran ayuda para el logro de los objetivos sanitarios para la década. En este sentido, evaluar la adherencia de los equipos de atención primaria a las terapias más efectivas, es de gran relevancia.

Nuestro estudio observó un alto cumplimiento en la prestación de la radiografía de tórax, hecho relevante considerando que esta prestación no se efectúa en los CAP, siendo realizado en centros radiológicos públicos o privados, dependiendo de la red de prestadores con que tiene convenios cada municipio. La calidad de los exámenes radiológicos será objeto de un análisis futuro. Sólo en $51,8 \%$ de los casos se realizó la consulta de confirmación antes de las $48 \mathrm{~h}$, hecho que debe llamarnos la atención, considerando el contexto en el cual se diagnostica este cuadro infeccioso. El control precoz es de vital importancia para evaluar la tolerancia a los medicamentos, confirmar el diagnóstico y conocer la evolución del cuadro infeccioso. Aun así, la administración precoz de antibióticos, se cumple en $94,8 \%$ de los casos estudiados, acción que ha demostrado ser uno de 
Tabla 3. C umplimiento del tratamiento antibiótico según G uía C línica AU GE

\begin{tabular}{|c|c|c|c|}
\hline & $\begin{array}{c}\text { H ombres } \\
n=35\end{array}$ & $\begin{array}{c}\text { M ujeres } \\
n=147\end{array}$ & $\begin{array}{c}\text { Total } \\
n=182\end{array}$ \\
\hline Tto Antibiótico № (\%) & $35(100)$ & $143(97,3)$ & $178(94,8)$ \\
\hline $\begin{array}{l}\text { Amoxicilina/ Ac clavulánico* } \\
\text { n (\%) } \\
\text { Días Tto atb mediana (IQR) }\end{array}$ & $\begin{array}{ll}20 & (57,1) \\
10 & (3)\end{array}$ & $\begin{array}{ll}89 & (62,2) \\
10 & (3)\end{array}$ & $\begin{aligned} 109 & (61,2) \\
10 & (3)\end{aligned}$ \\
\hline $\begin{array}{l}\text { Claritromicina* } \\
\text { n }(\%) \\
\text { Días Tto atb mediana (IQR) }\end{array}$ & $\begin{aligned} 4 & (11,4) \\
10 & (1,5)\end{aligned}$ & $\begin{array}{ll}27 & (18,9) \\
10 & (3)\end{array}$ & $\begin{array}{ll}31 & (17,4) \\
10 & (3)\end{array}$ \\
\hline $\begin{array}{l}\text { Eritromicina* } \\
\text { n }(\%) \\
\text { Días Tto atb mediana (IQR) }\end{array}$ & $\begin{array}{ll}2 & (5,7) \\
7 & (0)\end{array}$ & $\begin{array}{ll}6 & (4,2) \\
7 & (0)\end{array}$ & $\begin{array}{ll}8 & (4,5) \\
7 & (0)\end{array}$ \\
\hline $\begin{array}{l}\text { Amoxicilina } \\
\text { n (\%) } \\
\text { Días Tto atb mediana (IQR) }\end{array}$ & $\begin{array}{ll}8 & (22,9) \\
7 & (3)\end{array}$ & $\begin{array}{ll}17 & (11,9) \\
10 & (3)\end{array}$ & $\begin{aligned} 25 & (14,0) \\
7 & (3)\end{aligned}$ \\
\hline $\begin{array}{l}\text { Otros (Quinolonas/Penicilina) } \\
\text { n (\%) } \\
\text { Días Tto atb mediana (IQR) }\end{array}$ & $\begin{aligned} 1 & (2,8) \\
10 & (0)\end{aligned}$ & $\begin{array}{ll}4 & (2,8) \\
7 & (3)\end{array}$ & $\begin{array}{ll}5 & (2,8) \\
7 & (0)\end{array}$ \\
\hline $\begin{array}{l}\text { *Tto antibiótico ajustado a } \\
\text { Guía de Práctica Clínica } \\
\text { Uso Inhalador n (\%) } \\
\text { Otros medicamentos n (\%) }\end{array}$ & $\begin{array}{ll}26 & (74,3) \\
18 & (51,4) \\
18 & (51,4)\end{array}$ & $\begin{array}{l}122(85,3) \\
99(67,3) \\
97(66,0)\end{array}$ & $\begin{array}{l}148(83,1) \\
117(64,3) \\
115(63,2)\end{array}$ \\
\hline
\end{tabular}

Tabla 4. Uso de recursos profesionales en el tratamiento de la N AC

\begin{tabular}{|lccc|}
\hline & $\begin{array}{c}\text { H ombres } \\
\mathbf{n = 3 5}\end{array}$ & $\begin{array}{c}\text { M ujeres } \\
\mathbf{n = 1 4 7}\end{array}$ & $\begin{array}{c}\text { Total } \\
\mathbf{n = 1 8 2}\end{array}$ \\
\hline $\begin{array}{l}\text { Consulta médicas realizadas } \\
\text { 3 o más consultas \% }\end{array}$ & & & \\
$\begin{array}{l}\text { Consultas kinésicas } \\
\text { Indicado n (\%) }\end{array}$ & 28,1 & 46,1 & 42,8 \\
Mediana (IQR) & $13(37,1)$ & $60(40,8)$ & $73(40,1)$ \\
Rango & $2(2)$ & $2(2)$ & $2(2)$ \\
& $1-5$ & $1-7$ & $1-7$ \\
\hline
\end{tabular}

los factores más relevantes en la evolución y pronóstico de la enfermedad ${ }^{14,15}$.

Diversos estudios nacionales e internacionales ${ }^{16-18}$ demuestran que el agente causal más frecuente de la neumonía adquirida en la comunidad es el estreptococo pneumoniae. En este contexto destaca el alto nivel de estandarización en el uso de antibióticos al momento de tratar los 
cuadros de neumonías, siendo el tratamiento ajustado a la guía en $83,1 \%$.

El uso de amoxicilina (14\%), no está indicado en la Guía por la posibilidad de resistencia a los betalactámicos. Este hecho es controversial al considerar lo señalado por Soler et $\mathrm{al}^{19}$, que indica que el porcentaje de resistencia del estreptococo pneumoniae no supera $5 \%$. En cuanto al uso de inhaladores, en $92,3 \%$ de los casos se indicó salbutamol como primera elección, tal como lo recomienda la guía clínica. Las diferencias encontradas en la duración del tratamiento (119 días) se explican por la existencia de patologías crónicas respiratorias asociadas.

El buen desempeño de los profesionales de atención primaria en el manejo de la NAC, no sólo ha sido producto de la existencia de la guía clínica AUGE, sino de un trabajo que lleva más de 10 años de labor conjunta entre los equipos técnicos ministeriales, los servicios de salud y los profesionales que trabajan en el nivel primario de salud. Como se ha planteado a nivel internacional, los

\section{REFERENCIAS}

1. Martínez R, Reyes S, Lorenzo MJ, Menéndez R. Semin Respir Crit Care Med 2009; 30: 172-8.

2. Instituto Nacional de Estadísticas. Anuarios de Estadísticas Vitales, Chile, 2005.

3. VALDIVIA G. Epidemiología de la neumonía del adulto adquirida en la comunidad. Rev Chil Enf Respir 2005; 21: 73-80.

4. Ministerio de Salud Chile 2002. Los objetivos sanitarios para la década 2000-2010, $1^{\mathrm{a}}$ Edición, Ministerio de Salud, Santiago.

5. Ministerio de Salud Chile, Objetivos sanitarios para la década 2000-2010: Evaluación a mitad de periodo. Estado de Avance en los Objetivos de impacto, Subsecretaria de Salud Pública, División de Planificación Sanitaria, 2006, $1^{\text {a }}$ Edición, Ministerio de Salud, Santiago.

6. Ministerio de Salud Chile, Informe anual Programa de Prevención de enfermedades respiratorias 2009. Programa IRA/ERA, Subsecretaría de Redes Asistenciales, Ministerio de Salud, Santiago.

7. Letelier LM, Bedregal P. Health reform in Chile. Lancet 2006; 368: 2197-8.

8. Ministerio de Salud, Decreto № 170/2005, Garantías Explícitas del Régimen General de Garantías en Salud, Ministerio de Salud 2005. prestadores sanitarios demoran entre 5 a 8 años en cumplir las guías clínicas disponibles ${ }^{20}$, esto explicaría en parte el buen desempeño observado. Estrategias tan exitosas como la incorporación de salas de atención para enfermedades respiratorias del adulto (Salas ERA), la definición como grupo prioritario para la atención médica de los adultos mayores, la incorporación del profesional kinesiólogo al equipo de atención primaria y la provisión centralizada y estandarizada de medicamentos son elementos que han confluido en esta alta adherencia.

Nuestro próximo desafío en el proceso de reforma sanitaria, es cumplir a cabalidad con la garantía de calidad. La calidad de atención de los usuarios, no depende solamente de la existencia de guías clínicas, sino que debe ir acompañado de procesos intensivos de capacitación en terreno a los equipos locales de salud, como así también, de la implementación de metodologías simples y de bajo costo para evaluar periódicamente la red de prestadores y la satisfacción de los usuarios.

9. Fianders SA, Halm EA. Guidelines for communityacquired pneumonia. Are they reflected in practice? Treat Respir Med 2004; 3: 67-77.

10. GUTIÉRREZ F, MASIÁ M. Improving outcomes of elderly patients with community- acquired pneumonia. Drugs Aging 2008; 25: 585-610.

11. Rowe AK, de Savigny D, Lanata CF, Victora CG. How can we achieve and maintain high-quality performance of health workers in low-resource settings? Lancet 2005; 366: 1026-35.

12. Dangour AD, Albala C, Aedo C, Eibourne D, Grundy E, WALKER D, UAUY R. A factorial-design cluster randomized controlled trial investigating the costeffectiveness of a nutrition supplement and an exercise programme on pneumonia incidence, walking capacity and body mass index in older people living in Santiago, Chile: the CENEX study protocol. Nutr J 2007; 6: 14.

13. Ministerio de Salud. Guía Clínica Neumonía Adquirida en la Comunidad de Manejo Ambulatorio en persona de 65 años y más. $1^{\mathrm{a}}$ Edición Santiago, Ministerio de Salud, 2005.

14. Niederman MS, Brito V. Pneumonia in the older patient. Clin Chest Med 2007; 28: 751-71.

15. Brito V, Niederman MS. How can we improve the management and outcome of pneumonia in the elderly? Eur Respir J 2008; 32: 12-4. 
16. Bartlett JG, Mundy LM. Community-acquired pneumonia. N Engl J Med 1995; 333: 1618-24.

17. Henríquez A, González P, Cona E, Miranda P, Mendoza J, Giglio M ET AL. Diagnóstico etiológico de la neumonía del adulto: utilidad del lavado broncoalveolar. Rev Méd Chile 1991; 119: 758-64.

18. Díaz A, Calvo M, O’Brien A, Farías G, Mardónez JM, SALdías F. Utilidad clínica de los hemocultivos en pacientes hospitalizados por neumonía adquirida en la comunidad. Rev Méd Chile 2002; 130: 9931000.
19. Soler T, Salamanca L, Arbo G, Molina E. Estudio de sensibilidad in vitro de cepas de Streptococcus pneumoniae de infecciones respiratorias bajas en el Instituto Nacional del Tórax (INT). Rev Méd Chile 2002; 130: 304-8.

20. Shekelle PG, Ortiz E, Rhodes S, Morton SC, Eccles MP, Grimshaw JM, Woolf SH. Validity of the Agency for Healthcare Research and Quality clinical practice guidelines: how quickly do guidelines become outdated? JAMA 2001; 286: 1461-7. 